\title{
Flow with nanoparticle clustering controlled by optical forces in quartz glass nanoslits
}

\author{
Tetsuro Tsuji ${ }^{1,2}$ (C) Yuki Matsumoto ${ }^{2} \cdot$ Satoyuki Kawano $^{2}$
}

Received: 20 April 2019 / Accepted: 26 September 2019 / Published online: 17 October 2019

(c) The Author(s) 2019

\begin{abstract}
In this paper, we demonstrate nanoparticle flow control using an optical force in a confined nanospace. Using nanofabrication technologies, all-quartz-glass nanoslit channels with a sudden contraction are developed. Because the nanoslit height is comparable to the nanoparticle diameter, the motion of particles is restricted in the channel height direction, resulting in almost two-dimensional particle motion. The laser irradiates at the entrance of the sudden contraction channel, leading the trapped nanoparticles to form a cluster. As a result, the translocation of nanoparticles into the contraction channel is suppressed. Because the particle translocation restarts when the laser irradiation is stopped, we can control the nanoparticle flow into the contraction channel by switching the trapping and release of particles, realizing an intermittent flow of nanoparticles. Such a particle flow control technique in a confined nanospace is expected to improve the functions of nanofluidic devices by transporting a target material selectively to a desired location in the device.
\end{abstract}

\section{Introduction}

Nanofluidic devices are promising tools for the detection or identification of nano-materials (Sackmann et al. 2014). Recent developments in fabrication technologies allow us to design nanoscale channels or pores that have characteristic lengths comparable to the size of the targeted nanoparticles. Here, the targeted particles for engineered biomedical devices are biomolecules such as DNA (Tsutsui et al. 2010; Schneider et al. 2010; Merchant et al. 2010), viruses (Arima et al. 2018), or allergen particles (Kawaguchi et al. 2012). Using external forces, such as an electrophoretic force, these targets are guided to a narrow contraction in the nanofluidic device, where some detection or identification tools are installed. For instance, transverse nanogap electrodes were fabricated in a longitudinal nanochannel for the electrical detection of pollen allergen particles in Kawaguchi et al. (2012). Furthermore, such nanofluidics technologies are

Tetsuro Tsuji

tsuji.tetsuro.7x@kyoto-u.ac.jp

Satoyuki Kawano

kawano@me.es.osaka-u.ac.jp

1 Present Address: Graduate School of Informatics, Kyoto University, Kyoto, Japan

2 Graduate School of Engineering Science, Osaka University, Toyonaka, Japan expected to develop fast and low-cost nanopore sequencers to decode DNA, RNA, and peptides (Di Ventra and Taniguchi 2016). However, due to the smallness of the targets, their motion or flow are hard to control. To be more specific, the randomness inherent in Brownian motion becomes significant as the particle diameter decreases. Therefore, the development of manipulation techniques of such nanoscale targets in nanochannels is expected to improve the performance of the nanofluidic devices, providing a higher detection frequency and/or a finer signal resolution. In recent years, some efforts have been made to control the translocation of particles (Yusko et al. 2011; Keyser 2011; Tanaka et al. 2016). However, complicated fabrication processes of nanofluidic devices are necessary for these approaches, and the development of feasible methods is expected to expand the usefulness of nanofluidic devices to various research fields. The present paper aims at establishing a novel and feasible experimental protocol to control the particle transport in nanospace.

Ever since optical tweezers were reported by Ashkin (1970), forces exerted by light have attracted much attention in biosciences, in which the manipulation of microobjects, such as cells, is important. The optical trapping was also used in a nanopore technology to investigate the force acting on the DNA in a nanopore (Keyser et al. 2006). In Keyser et al. (2006), the DNA was attached to a bead with $2 \mu \mathrm{m}$ diameter, which was optically trapped and controlled. The 
advantage of the use of an optical force comes from the fact that it is a non-contact method without sophisticated and complicated fabrication processes. The theoretical model of optical forces varies according to the ratio between the particle diameter $d$ and the wavelength of light $\lambda_{\mathrm{L}}$ : we use ray optics for $d \gg \lambda_{\mathrm{L}}$ (Ashkin et al. 1986), the Lorentz-Mie theory for $d \sim \lambda_{\mathrm{L}}$, and Rayleigh theory for $d \ll \lambda_{\mathrm{L}}$ (Harada and Asakura 1996). Since the laser with the wavelength of $\lambda_{\mathrm{L}}=1064 \mathrm{~nm}$ is frequently used due to its low absorption to aqueous solutions, microparticles with diameter $d>\lambda_{\mathrm{L}}$ are usually described by ray optics (Nito et al. 2018) and nanoparticles with diameter $d<\lambda_{\mathrm{L}}$ are by the Lorentz-Mie or Rayleigh theory (Harada and Asakura 1996; Nagura et al. 2019).

In these days, the optical trapping of microparticles is well established technique and even commercialized. However, as the size of the targets shrinks, the optical force acting on particles decreases rapidly (Harada and Asakura 1996; Nito et al. 2018) and the Brownian motion becomes more prominent. These matters make the nanoparticle optical trapping difficult. If we can extend the size range of the targets in optical trapping to nanoscale, not only cells with micrometer scales, but also viruses and biomolecules, which have equivalent diameters of tens or hundreds of nanometers (Briggs et al. 2006; Bouvier and Palese 2008), are also within the scope of application of optical trapping. Therefore, the optical manipulation of nanoparticles is a challenging and promising research area, but has not yet been systematically investigated for the application to engineering fields. In this paper, having in mind the application to nanoscale targets in nanofluidic devices, we propose to use optical forces to control the clustering and flow of nanoparticles.

We use the so-called nanoslit as the nanofluidic device, where the channel height is uniform and nanoscale. To effectively use the optical force for particle flow control, we propose the following strategy. The optical force is the force acting on micro- and nano-materials induced by their irradiation by light. In practice, we use a focused laser to increase the power density to manipulate the targets. Because the effective spatial range of optical forces is limited to $O(1) \mu \mathrm{m}$, we use a contraction channel, which has been widely used in micro- and nanofluidic devices (Tsutsui et al. 2010; Schneider et al. 2010; Merchant et al. 2010; Arima et al. 2018; Kawaguchi et al. 2012), as a test section. To be more precise, two wide channels connect a narrow channel, which we call the contraction. This geometry may be considered as inplane "nanopore". In this way, all the particles targeted for translocation into the contraction are subject to the effect of optical forces and trapped. When we stop the laser irradiation, the trapped particles are released and flow into the contraction. That is, we can control the onset of nanoparticle translocation into the narrow part by switching trapping and release. In the state-of-the-art nanofluidic devices such as nanopore sequencer (Tsutsui et al. 2010; Di Ventra and Taniguchi 2016), the narrow part may have the function for detection and/or identification of translocating target objects. Therefore, we can selectively transport the targets to this sensing part using the proposed method, and choose a desired translocation speed and frequency. It is important and should be remarked that we restrict the motion of targets in the direction of beam propagation. This is realized using a nanoslit with nanoscale dimensions in the beam propagation direction. With the aid of such a restriction, we can stably trap the target particles without pushing them away. In addition, the nanoslit confinement can diminish unwanted thermal and fluid phenomena, such as thermal convection and thermophoresis in microfluidic systems (Tsuji et al. 2017, 2018). Therefore, the proposed device in the present study is a suitable starting point for investigating the optical manipulation in the nanofluidic devices and developing novel particle flow control techniques.

\section{Experimental method}

In this section, we first describe and characterize the nanofluidic device developed in the present paper in Sect. 2.1. The method of fabrication is given in detail in Sect. 2.2, since the bonding process of two quartz glass substrates in this paper is rather new technique. Then, we present in Sect. 2.3 the overall optical setup, which is a fluorescent microscope combined with an optical trapping apparatus. Section 2.4 is devoted to describe the experimental procedure and the detail of sample solution tested in the present paper. Some remarks on optical forces acting on nanoparticles are mentioned in Sect. 2.5. Finally, surface effects are introduced in Sect. 2.6.

\subsection{Description of the device}

Fabrication of microchannels using polydimethylsiloxane (PDMS) is widely applied in the field of microfluidics. In this fabrication method, the PDMS block is cast from a master mold with a channel pattern and is bonded to a substrate to form microchannels. However, when the channel height is nanoscale, any slight deformation of the PDMS block leads to the collapse of the nanochannel, that is, the PDMS roof easily sticks to the bottom. Moreover, the PDMS channel can be deformed by laser irradiation, as reported in Fukuyama et al. (2018). Therefore, the conventional fabrication method using PDMS may not be suitable for our experiments, where the laser irradiates the nanochannels. To overcome such difficulties, we fabricate nanochannels consisting of quartz glass substrates without PDMS. The fabrication process is described in Sect. 2.2, and here, we focus on the description of the device. 
(a) Top view Nanoslit pattern

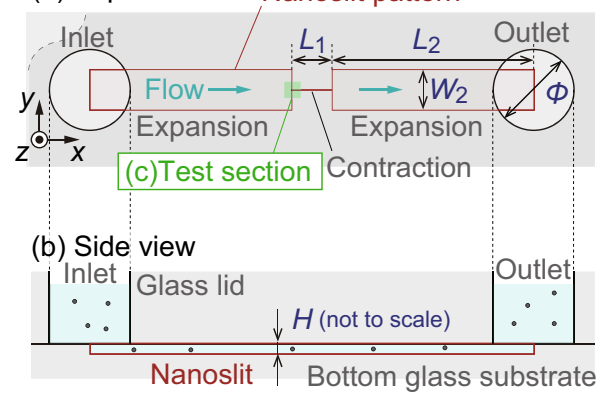

(c) Test section in $x y$-plane

(d) Test section

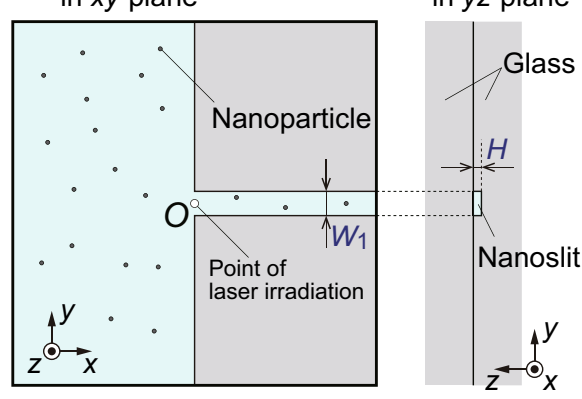

Fig. 1 Overview of the experiments. a Top and $\mathbf{b}$ side views of the device, where two glass substrates are bonded to each other to form the nanoslit shown in $\mathbf{b}$. The bottom glass substrate has the dryetched pattern shown in a, which has a nanoscale height $H=635 \mathrm{~nm}$ as shown in $\mathbf{b}$. The pattern has two expansion channels connected by a single contraction channel. The top glass substrate, that is, the lid, has two holes with a diameter $\Phi=2 \mathrm{~mm}$ to provide inlet and outlet, as shown in $\mathbf{b}$, where fluid flow is in the positive $x$ direction. $\mathbf{c}$ The test section in the $x y$-plane, which is the region near the entrance of the contraction channel shown in a. The laser irradiates the center of the test section. $\mathbf{d}$ Test section in the $y z$-plane

An overview of the device is provided in Fig. 1a-d. Figure 1a, $\mathrm{b}$ shows the top and side views of the device, respectively. The device has a nanoslit channel, which consists of three parts, as shown in Fig. 1a. Namely, the nanoslit pattern has two expansion channels bridged by a single contraction channel. These two expansion channels are connected to inlet and outlet reservoirs with diameter $\Phi$, and the flow of sample solution is induced in the positive $x$ direction. The dimensions in Fig. 1a are set as $L_{1}=1 \mathrm{~mm}, L_{2}=5 \mathrm{~mm}$, and $W_{2}=1 \mathrm{~mm}$. The width of the contraction $W_{1}$, which is shown in Fig. 1c, is set as $W_{1}=5 \mu \mathrm{m}$. The channel height $H$ shown in Fig. $1 \mathrm{~b}$ (not to scale) is $635 \pm 10 \mathrm{~nm}$, which is measured by a laser-scanning digital microscope (OLS4100, Olympus, Tokyo, Japan). It should be emphasized that the nanoslit is produced by bonding two quartz glass substrates without PDMS, as shown in Fig. $1 \mathrm{~b}$ and described in Sect. 2.2. Some recent progress on the fabrication of glass-made nanochannels is summarized in a review article (Xu 2017).

Figure 1c shows a magnified view of the test section near the entrance of the contraction channel, as shown in
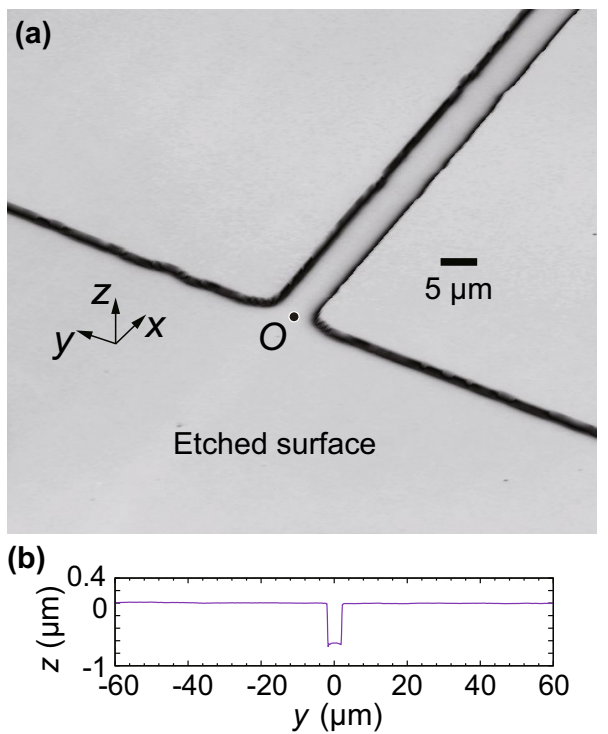

Fig. 2 a 3D-reconstructed image from the measurement of the height distribution. b Height profile in the cross section at $x=12 \mu \mathrm{m}$. We measure the height $H 30$ times along the $x$ direction in the contraction channel, and obtain $H=635 \pm 10 \mathrm{~nm}$. The variation of $H$ is less than $2 \%$ and we can conclude that the fabrication of the nanoslit with the contraction channel is carried out with high precision

Fig. 1a. The entrance, that is, the center of the test section, is irradiated with a near-infrared laser with a wavelength of $1064 \mathrm{~nm}$. This wavelength is standard and frequently used, e.g., in biosciences, to avoid the absorption of the laser by water solutions. Nanoparticles are transported in the positive $x$ direction and flow into the contraction. Therefore, all the nanoparticles translocating the contraction are subject to the laser. Figure $2 \mathrm{a}$ shows a $3 \mathrm{D}$ reconstruction of the measured height data $H$ using the laser-scanning digital microscope. The concave part is fabricated using reactive-ion etching (RIE-10NR, Samco, Kyoto, Japan). Figure $2 b$ shows the cross-sectional height profile $H$ at $x=12 \mu \mathrm{m}$.

\subsection{Fabrication of the device}

\subsubsection{Reactive-ion etching of bottom quartz glass substrate}

The fabrication of the bottom glass substrate in Fig. 1a is summarized in Fig. 3. (a) A quartz glass substrate $\left(25 \times 25 \times 0.5 \mathrm{t} \mathrm{mm}^{3}\right.$, Labo-USQ, Daico Mfg, Kyoto, Japan $)$ is sonicated successively in acetone, ethanol, and ultra-pure water for $15 \mathrm{~min}$ each. The substrate is then dried by heating at $200^{\circ}$ for $5 \mathrm{~min}$. (b) A positive photoresist (AZ5214E, Merck, Darmstadt, Germany) is spin-coated onto the substrate, and a prebake process for $10 \mathrm{~min}$ at $80^{\circ}$ is carried out. (c) The substrate is exposed to ultra-violet light at $20 \mathrm{~mJ} \mathrm{~cm}^{-2}$ thorough a photomask with the nanoslit pattern, 

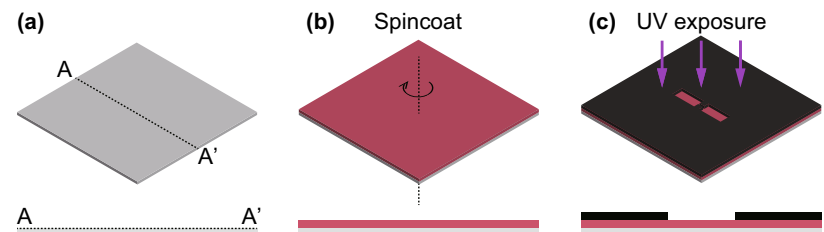

(d)

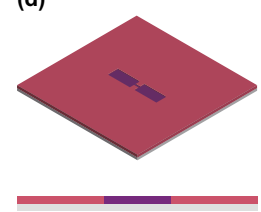

(e) Development

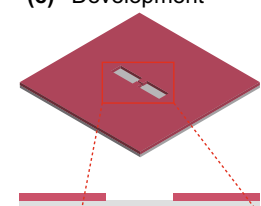

(f) Reactive-ion etching
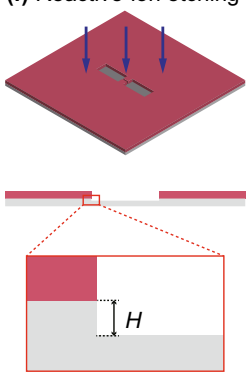

(g) Remove resist
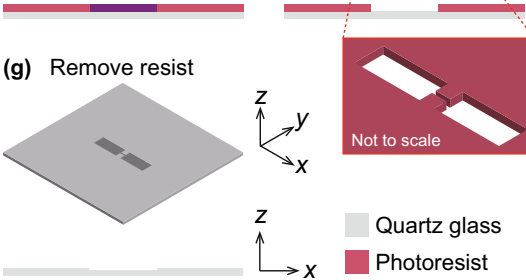

Photomask

Exposed photoresist

Fig. 3 Process for fabricating the nanoslit channel using photolithography. a Cleaned quartz glass. b Spin-coating of a photoresist. c Ultra-violet exposure through a photomask. d Removal of the photomask. e Development. f Reactive-ion-etching. g Removal of the photoresist

as described in Fig. 1a. (d) The photomask is removed. (e) The substrate is immersed in a developer solution (a 1:1 mixture of ultra-pure water and AZ developer, Merck, Darmstadt, Germany) for 1 min to obtain the resist mask for the nanoslit pattern. (f) Reactive-ion etching (RIE-10NR, Samco, Kyoto, Japan) is carried out for $30 \mathrm{~min}$ using $\mathrm{CHF}_{3}$ as a process gas. Process pressure and the power are set to $4 \mathrm{~Pa}$ and $100 \mathrm{~W}$, respectively. (g) The resist mask is removed by sonicating the substrate successively by developer, acetone, ethanol, and ultra-pure water for 15 min each. Any tiny residues on the substrate result in the failure of the bonding process, as described in Sect. 2.2.3. Therefore, the cleaning process is important and must be carried out carefully. When any residues on the substrate are found by a microscope observation, we immerse the substrate in acetone and scrub it with a cotton swab until the residues are removed.

\subsubsection{Ultrasonic drilling of a quartz glass lid}

We use an ultrasonic polisher (Takumix HD, Sea Force, Tokyo, Japan) and a diamond powder (DK-400, As One, Osaka, Japan) to fabricate two holes for the inlet and outlet on the quartz glass lid, as shown in Fig. 1a, b. The powder is dispersed in a solution, which is dripped onto the glass lid. We push the polisher through the dripped solution until the polisher penetrates the glass lid. Residual powder leads to the failure of the bonding process in Sect. 2.2.3 and it must be removed with special attention. After the above drilling

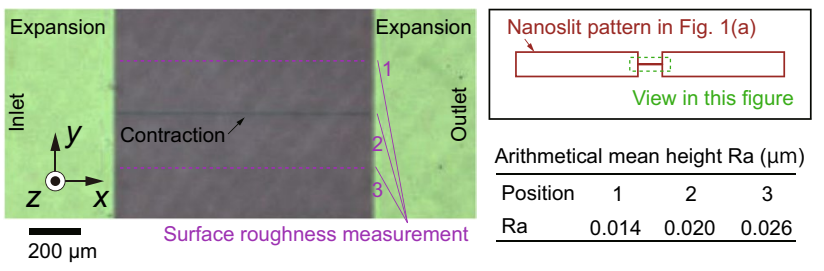

Fig. 4 Photograph of the nanoslit after the bonding process. The observed color of the nanoslit, which may be due to optical interference, indicates successful nanoslit formation. Before the bonding, surface roughness measurement on the etched surface of the bottom quartz glass substrate is carried out along $x$ direction for $y=0 \mu \mathrm{m}$ (in the contraction) and $y= \pm 200 \mu \mathrm{m}$, as indicated in the figure. Measured arithmetical mean height $\mathrm{Ra}(\mu \mathrm{m})$ is given in the table

process, we first wash the glass lid using a neutral detergent. Then, we sonicate the glass lid for $5 \mathrm{~min}$ in acetone, $5 \mathrm{~min}$ in ultra-pure water, $15 \min$ in $\mathrm{NaOH}$ aqueous solution of $0.1 \mathrm{M}$ concentration, and $10 \mathrm{~min}$ in ultra-pure water. It should be noted that the residual powder may scratch the glass lid. This scar can cause a micro-scale roughness on the glass, which may lead to the failure of the bonding process, as described in Sect. 2.2.3. The use of $\mathrm{NaOH}$ solution in the above cleaning process is necessary to remove the powders gently.

\subsubsection{Bonding of two quartz glasses using vacuum ultra-violet light}

The quartz glasses fabricated in Sects. 2.2.1 and 2.2.2 are bonded each other to make the nanoslit channel, as introduced in Sect. 2.1. Before the bonding process, we use a laser-scanning digital microscope to confirm carefully that no residue is left on the bottom and lid glasses. The glasses are placed in a nitrogen atmosphere and exposed to a vacuum ultra-violet (VUV) light (wavelength $172 \mathrm{~nm}$, $10 \mathrm{~mW} \mathrm{~cm}{ }^{-2}$, SUS06, Ushio, Tokyo, Japan) for $100 \mathrm{~s}$. After the exposure, the treated surfaces of the glasses are contacted and pushed together gently by hand. If the bonding succeeds, we can observe a colored nanoslit pattern using a microscope, as shown in Fig. 4; this color may be attributed to optical interference in the nanoslit. If the bonding process fails, the multicolored interference stripes are observed instead of the uniform colored region, as shown in Fig. 4. In such cases, one should repeat the cleaning processes in Sects. 2.2.1 and 2.2.2. Such a bonding technique using VUV light is also recently reported as a facile method (Wang et al. 2019).

\subsection{Optical setup}

Figure 5 shows the optical setup in the present experiment. A near-infrared laser (Fitel ASF1JE01, Furukawa Electric, Tokyo, Japan) with wavelength $1064 \mathrm{~nm}$ is expanded 


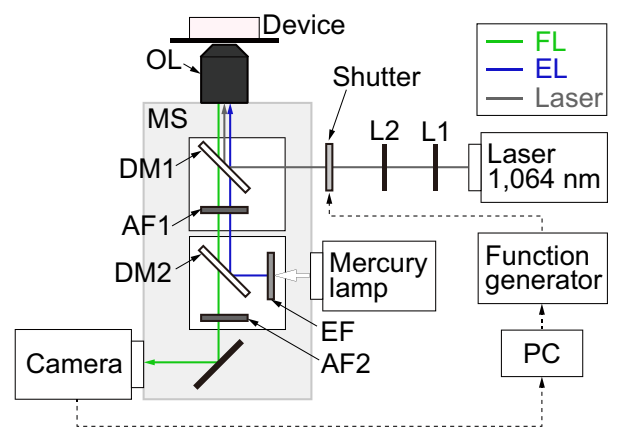

Fig. 5 Optical setup of the experiment. A near-infrared laser with wavelength $1064 \mathrm{~nm}$ is irradiated to the device. L1, L2 collimating lenses, $O L 50 \times$ objective lens with numerical aperture $0.65, D M 1$, $D M 2$ dichroic mirrors, $A F 1, A F 2$ absorption filters, $E F$ excitation filter, $F L$ fluorescent light, $E L$ excitation light, $M S$ inverted microscope, $P C$ personal computer

and collimated using lenses L1 and L2 and guided into an inverted microscope (IX-73, Olympus, Tokyo, Japan). The laser irradiation of the device is controlled by a mechanical shutter (SSH-25RA-W; SSH-C2B, Sigmakoki, Tokyo, Japan) using a function generator (WF1973, NF, Kanagawa, Japan). The shutter is synchronized with the image acquisition of an sCMOS high-speed camera (Zyla 5.5, Andor Technology, Tokyo, Japan). The laser irradiates the nanoslit device through an objective lens $(50 \times$, numerical aperture $=$ 0.65, LCPLN50XIR, Olympus, Tokyo, Japan). The transmittance of the laser through the whole optical system is $30.4 \%$, which is measured with a laser power meter (3A-QUAD, Ophir, Saitama, Japan). The laser power is set to $2.0 \mathrm{~W}$ at the laser source, and thus, the effective power delivered to the device can be estimated as $608 \mathrm{~mW}$. The size of the laser irradiation spot on the device is estimated as $4.4 \mu \mathrm{m}$. The spot size is determined as the diameter at which the laser intensity becomes $1 / e^{2}=0.135$ of the maximum. The temperature increase due to the laser irradiation with a similar optical setup used in our previous study (Nito et al. 2018) was confirmed to be $1 \mathrm{~K}$, and we consider the effect of temperature rise to be negligible. An excitation light is generated from a mercury lamp (U-HGLGPS, Olympus, Tokyo, Japan) thorough an excitation filter and irradiates the sample solution in the nanoslit device. Fluorescence from the sample is monitored using the camera and recorded in a personal computer.

\subsection{Experimental procedure}

Experiments are conducted at room temperature, that is, $297 \pm 0.3 \mathrm{~K}$. We use a polystyrene (PS) nanoparticle dispersion as a sample solution. The diameter of the nanoparticles is denoted by $d$, and in this study, we use particles with $d=490 \mathrm{~nm}$ (F8812, red fluorescent, carboxylate-modified,
Lot No. 1892319, $d=490 \pm 15$ nm, Molecular Probes, Eugene, USA) or $d=190 \mathrm{~nm}$ (F8811, yellow-green fluorescent, carboxylate-modified, Lot No. 1927586, $d=190 \pm 14 \mathrm{~nm}$, Molecular Probes, Eugene, USA). A working fluid is prepared by diluting the nanoparticle dispersion with the mixture of a buffer (Tris- $\mathrm{HCl}, 10 \mathrm{mM}, \mathrm{pH}$ 8.0, Nippon Gene, Tokyo, Japan) and a non-ionic surfactant (Triton X-100, 0.5 vol\%, MP Biomedicals, Santa Ana, California, USA). The buffer is used to stabilize the $\mathrm{pH}$ of the sample solution and the surfactant to avoid the adsorption of nanoparticles onto the channel walls. The addition of the buffer and the surfactant in the solution results in a good dispersal state of the particles, which do not stick to the inner walls over the entire nanoslit. The inner walls of the nanoslit device are hydrophilized by a corona treater (BD20AC, Electro-Technic Products, Chicago, USA) to facilitate the filling of the nanoslit with solution. Because the water levels of the two reservoirs in Fig. $1 \mathrm{~b}$ are different, a net flow is induced in the nanoslit channel. In this paper, we control the flow rate, so that the flow speed in the contraction channel is less than $100 \mu \mathrm{m} \mathrm{s}^{-1}$, which is suitable for image acquisition with our optical setup. Here, the flow speed is estimated from the translocating particle motion without laser irradiation. Since the nanoparticle size is comparable to the nanoslit height, these particles may not be ideal tracer. Nonetheless, we expect that the order of flow speed can be estimated by this method.

Note that we attach a PDMS block to the glass lid in Fig. 1b. The PDMS block has two holes, which are located at the same positions of the inlet and outlet of the glass lid. The attachment of the PDMS is necessary to increase the volume of working fluid in the inlet and outlet to a sufficient level; otherwise, the device may dry out during the experiment.

\subsection{Remark on optical forces}

Before going into details about the results, we describe the optical forces acting on the particles. The laser used in this study is considered as a Gaussian beam, which has an intensity profile in a plane perpendicular to the direction of beam propagation. By neglecting the absorption of light by the particles, the optical forces acting on the particles can be divided into two contributions: scattering and gradient forces. For the Gaussian beam used in this study, the scattering force acts in the beam propagation direction, that is, the positive $z$ direction. The gradient force mainly acts in the negative radial direction $r\left(=\sqrt{x^{2}+y^{2}}\right)$. That is, the gradient force acts as optical tweezers to fix the particles at the focal spot. In our experiments, the particles are confined in a nanoslit with a height $H=635 \mathrm{~nm}$ in the $z$ direction. Therefore, we expect that the scattering force in the $z$ direction does not play a significant role in the particle motion for the case of $d=490 \mathrm{~nm}$, and only the gradient forces should be taken into account. On the 
other hand, for the case of $d=190 \mathrm{~nm}$, scattering force pushes the particles in the $z$ direction, that is, the optically trapped particles stay close to the lid of the channel, as in the previous study (Nito et al. 2018).

\subsection{Electrostatic properties and surfactant}

As will be seen in Sect. 3.1, we have a good dispersion state of nanoparticles in our experiments. Concerning this point, we comment on the effects of electric double layers (EDLs) and the surfactant as follows.

The surfaces of nanoparticles are carboxylate-modified, that is, the particles are negatively charged and their zeta potential may be different from those without surface modification. In our previous study (Tsuji et al. 2018), we measured the zeta potential of carboxylate-modified PS particles using an electrophoretic light-scattering spectrophotometer (ELS-6000, Otsuka Electronics, Japan). The zeta potential $\zeta$ for $d=1 \mu \mathrm{m}$ PS particles in the mixture of Tris- $\mathrm{HCl}$ and Trition X-100 was $\zeta=-36 \pm 2 \mathrm{mV}$. It should be remarked that Triton X-100 reduces the magnitude of zeta potential. In fact, $\zeta=-80 \pm 3 \mathrm{mV}$ was obtained without Triton X-100. In addition, we also measured the zeta potential of $\mathrm{SiO}_{2}$ (silica) particles without any surface modification in the mixture of Tris- $\mathrm{HCl}$ and Trition X-100, which was $\zeta=-19 \pm 2 \mathrm{mV}$. Therefore, we suppose that the surface of the quartz glass inner walls in the present study is also negatively charged. As a result, EDLs are formed at the liquid-solid surfaces of the particles and the inner walls, where the thickness of the EDLs is characterized by a Debye length estimated as $\lambda_{\mathrm{D}}=9.7 \mathrm{~nm}$ (Nguyen and Wereley 2002). Since Triton $\mathrm{X}-100$ is a non-ionic surfactant, the effect of Triton X-100 is not considered in the above calculation of EDL.

Next, the effect of Triton X-100 is considered. It was reported that the micelle size of Triton X-100 is $4 \mathrm{~nm}$ at room temperature (Streletzky and Phillies 1995). If the surfaces of particle and channel are covered with the molecules of Triton X-100, the thickness of the surfactant layer may be considered as $\lambda_{\mathrm{T}}=2 \mathrm{~nm}$. Therefore, the thickness of the EDL $\lambda_{\mathrm{D}} \approx 10 \mathrm{~nm}$ plus that of the surfactant $\lambda_{\mathrm{T}}=2 \mathrm{~nm}$ results in the situation, where the particle and inner wall surfaces are covered with a layer of thickness approximately $\lambda=12 \mathrm{~nm}$. The particles thus tend to stand off from the inner walls at the distance at most $2 \lambda=24 \mathrm{~nm}$. The presence of the layer with thickness $\lambda=12 \mathrm{~nm}$ is expected to give a good dispersion state, and its effect on the clustering behavior will be discussed later in Sect. 3.1.

\section{Results and discussion}

We show the results for particle diameter $d=490 \mathrm{~nm}$ and $d=190 \mathrm{~nm}$ in Sects. 3.1 and 3.2, respectively. The former case will produce a two-dimensional clustering, while the latter a three-dimensional one.

\subsection{Two-dimensional clustering in nanoslit}

In this section, we present the results of nanoparticles with diameter $d=490 \mathrm{~nm}$. The concentration of the particles in the working fluid is $10^{-2} \mathrm{wt} \%$. The focused laser irradiates the entrance of the contraction, as shown in Fig. 1c. We use a mirror unit (U-FGW, Olympus, Tokyo, Japan) designed to observe the fluorescence of the particles with $d=490 \mathrm{~nm}$ (excitation: $580 \mathrm{~nm}$; emission: $605 \mathrm{~nm}$ ).

The $d=490 \mathrm{~nm}$ particles are expected to yield a two-dimensional cluster in the nanoslit. In our previous study (Nito et al. 2018), where the channel height was $H=7.0 \mu \mathrm{m}$ and $d=2.93 \mu \mathrm{m}$, particles formed a twodimensional cluster with a hexagonal packing state at the solid-liquid interface, that is, the roof of the channel, due to the gradient forces. In Nito et al. (2018), only two particles could stack in the $z$ direction because $H / d \approx 2.39$. The device in the present study has a much smaller height-todiameter ratio $H / d \approx 1.30$ than $H / d \approx 2.39$ in Nito et al. (2018), and we expect a similar two-dimensional clustering behavior with only one layer of particles.

Figure 6 shows the time series of snapshots, where the laser irradiation starts at $t=10 \mathrm{~s}$. The dashed lines in Fig. 6 indicate the outline of the nanoslit pattern. In panel (a) at $t=30 \mathrm{~s}$, one trapped particle is observed. As time goes on, the number of trapped particles $N$ increases and a cluster is formed. For the particles with $d=490 \mathrm{~nm}$, it is possible to distinguish each particle, and thus, we count the number of trapped particles by carefully looking at the video frame-byframe. During the laser irradiation, no particle enters into the contraction channel, and the number of particles in the cluster reaches $N=43$ at $t=300 \mathrm{~s}$. The longer time experiment is supposed to increase the cluster size. However, we suspect that the size will saturate at a certain value, because the size of the laser spot is limited and some particle will not be trapped eventually.

Figure $7 \mathrm{a}$ shows the time development of the number of particles $N$ in the cluster, where the insets present the magnifications of corresponding snapshots in Fig. 6. We define an equivalent diameter $d_{N}$, as shown in Fig. 7b, to evaluate the cluster as follows. In two dimensions, the hexagonal arrangement of circles is known to yield the highest packing density factor of $\rho=0.9069$. One may suspect that the cluster takes the three-dimensional packing, as shown in Fig. 7c. However, the two-dimensional projection 

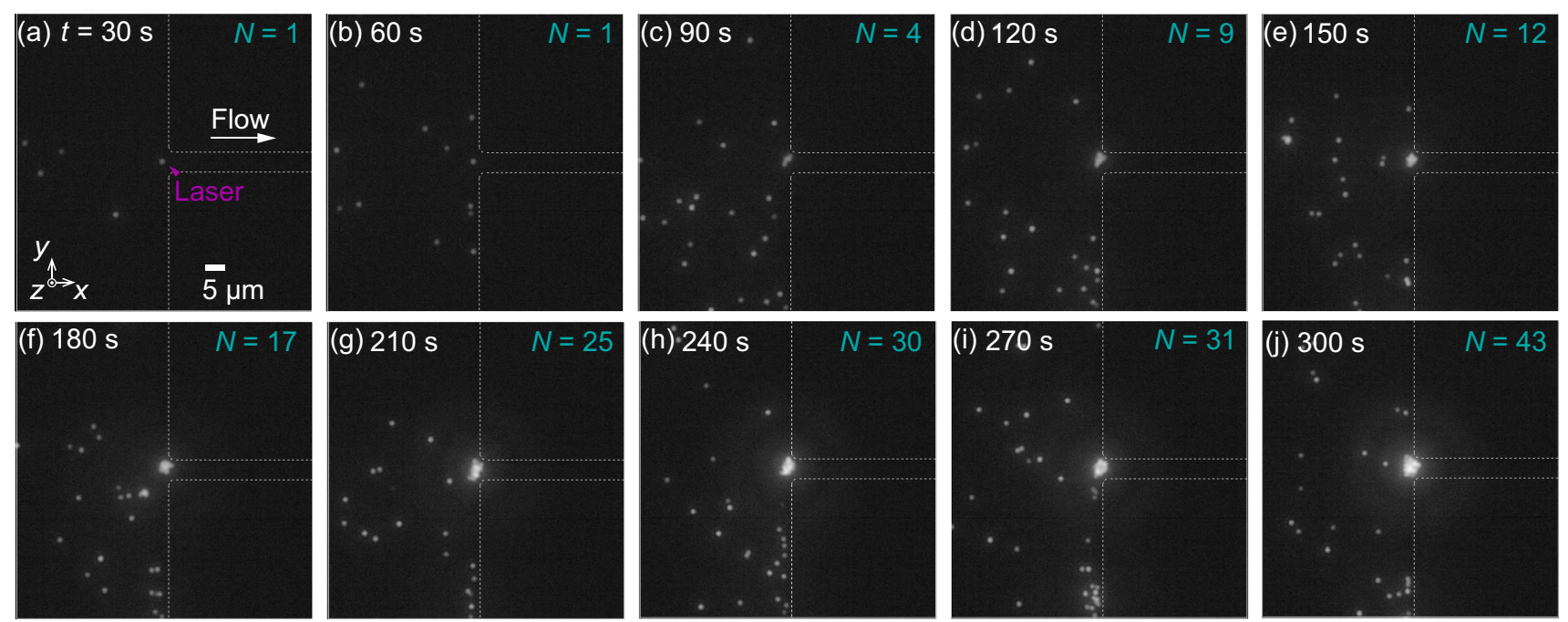

Fig. 6 Time series of the snapshots for the case of particles with $d=490 \mathrm{~nm}$ at $t=\mathbf{a} 30$, b 60 , c 90, d 120 , e 150, f 180, g 210, h $240, \mathbf{i} 270, \mathbf{j} 300 \mathrm{~s}$. The laser irradiation is incident at the center of

(a)

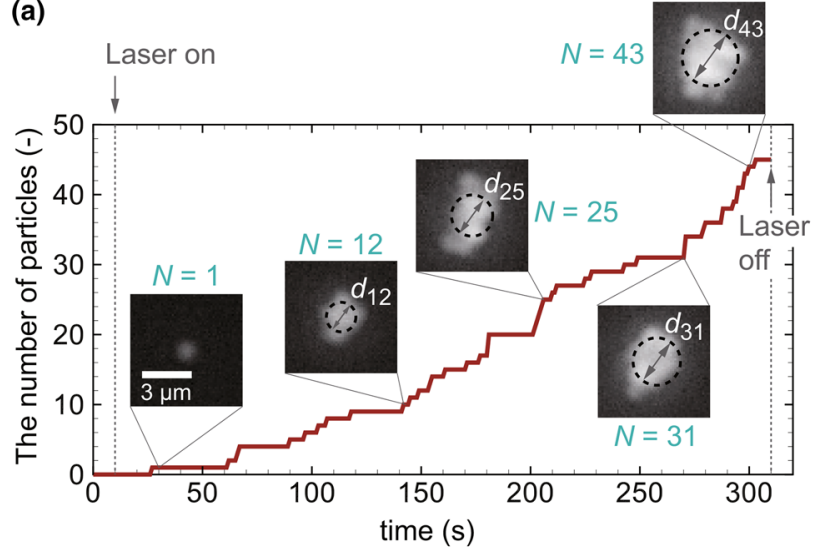

(b) Equivalent diameter $d_{N}$

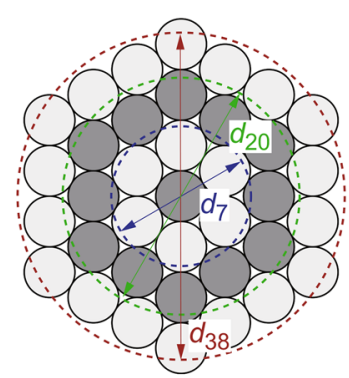

(c)

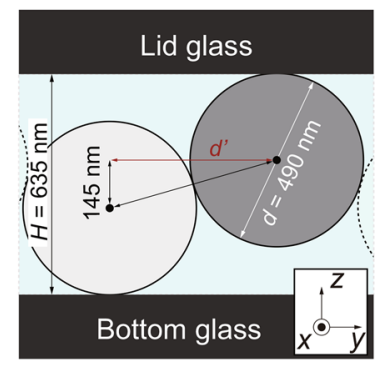

Fig. 7 a Time development of the number of particles $N$ in the cluster for the case of $d=490 \mathrm{~nm}$. b Equivalent diameter $d_{N}$ of the cluster is indicated by a dashed circle in each inset. c Schematic of a threedimensional closed packing state. The effective distance $d^{\prime}=468 \mathrm{~nm}$ is the two-dimensional projection of the inter-particle distance onto the $x y$-plane

$d^{\prime}=468 \mathrm{~nm}$ in the figure is almost the same as the interparticle distance $d=490 \mathrm{~nm}$. Moreover, the scattering force each image, as shown in (a). The dashed lines show the outline of the nanoslit pattern. A cluster of particles formes and grows as time goes on

pushes the particle to the positive $z$ direction, and we expect the less possibility of having the configuration, as described in Fig. 7c. For these reasons, we do not have to discuss the structure in the $z$ direction. Therefore, the two-dimensional cluster of $N$ particles has an equivalent area $A=\pi(d / 2)^{2} / \rho$, and thus, an equivalent diameter $d_{N}=2 \sqrt{(A / \pi)}$. The dashed circles in Fig. $7 \mathrm{~b}$ indicate circles with diameters $d_{N}$ with $N=7,20$, and 38 for reference. It should be noted that the equivalent diameter $d_{N}$ in Fig. 7a is slightly smaller than the observed cluster. This under-estimation may be attributed to the fact that fluorescing particles look larger than their actual sizes. Another possibility for the under-estimation is the presence of the layer with thickness $\lambda=12 \mathrm{~nm}$ introduced in Sect. 2.6. To consider the effect of this additional layer, we replace $d=490 \mathrm{~nm}$ by $d_{*}=490+2 \lambda=514 \mathrm{~nm}$ and $h=635 \mathrm{~nm}$ by $h_{*}=635-2 \lambda=611 \mathrm{~nm}$. In this case, $d^{\prime}$ in Fig. $7 \mathrm{c}$ is replaced by $d_{*}^{\prime}=505 \mathrm{~nm} \approx d_{*}$, and we can again neglect the three-dimensional configuration. Equivalent diameter of the cluster $d_{N}$ is replaced by $d_{N *}$, where $d_{N *} / d_{N} \approx 1.05$. Therefore, if we consider the presence of the layer with thickness $\lambda=12 \mathrm{~nm}$, the cluster size becomes $5 \%$ larger than that without the layer. In any case, we can conclude that the particles are confined in a one-layer cluster, because $d_{N}$ would overestimate the cluster size if the cluster consists of more than two layers.

Figure 8 shows the nanoparticle flow into the contraction channel. Note that the particles are not ideal tracer in our experiments and the particle motion does not quantitatively represents the flow of continuous phase. In the figure, we stop the laser irradiation at $t=0 \mathrm{~s}$, and the subsequent particle flow is displayed. It is seen that the cluster expands into a cloud of nanoparticles and the speed of the cloud 


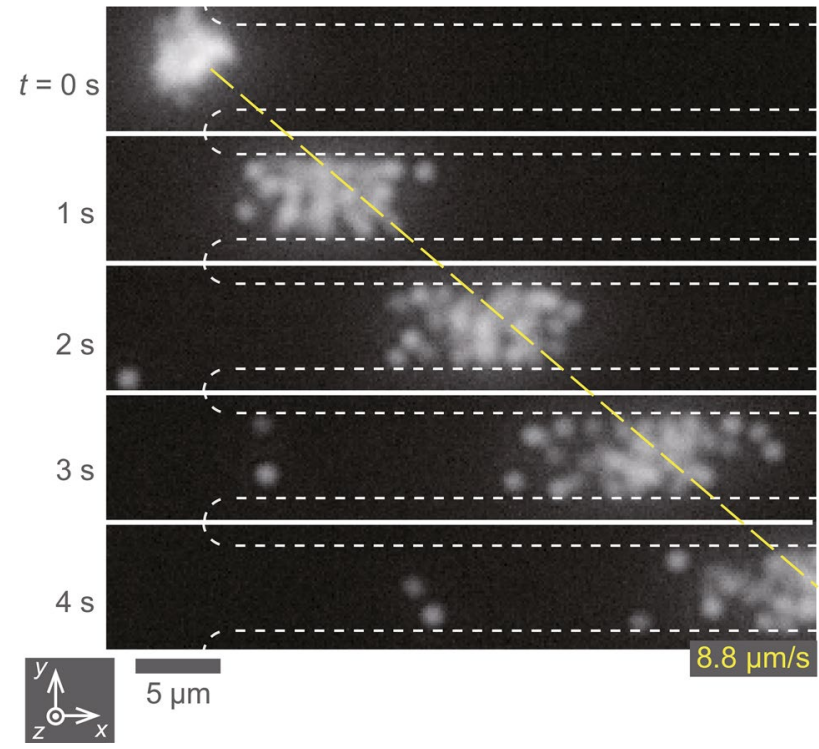

Fig. 8 Time series of the release of the cluster for $d=490 \mathrm{~nm}$. The laser irradiation is stopped at $t=0 \mathrm{~s}$, and the particles flow into the channel as time goes on. The yellow dashed line indicates the particle flow speed of $8.8 \mu \mathrm{m} / \mathrm{s}$

can be estimated as $8.8 \mu \mathrm{m} \mathrm{s}^{-1}$. It should be remarked that the particle motion indicates a plug-like particle flow for the $y$ direction in the contraction channel, which means that hydrodynamic inertial effects from the walls at $y= \pm W_{1} / 2$ (Amini et al. 2014; Martel and Toner 2014) are insignificant. The plug-like flow is also confirmed using the particle image velocimetry. That is, the $x$ component of the particle flow velocity is uniform in the $y$ direction with a standard deviation less than $8 \%$. Because the Poiseuille flow in a thin rectangular channel is considered as plug-like (Bruus 2007) in the $y$ direction away from the inner walls, the observed plug-like particle flow is also theoretically reasonable. On the contrary, the flow profile of continuous phase in the $z$ direction is Poiseuille-like (Bruus 2007). This is confirmed from the snapshots in Fig. 8, where the cluster length in the $x$ direction is elongated as the cluster goes downstream. More specifically, the particles close to the inner walls at $z= \pm H / 2$ are slower and those at $z \approx 0 \mu \mathrm{m}$ are faster. We note that this type of configuration of dispersed phases may be connected with the biological flow of red blood cells (RBCs). In the visualization experiments of such biological flows (Kikuchi and Mochizuki 2011), the RBCs flowed at the center of microchannels.

It should be emphasized that the particles enter into the contraction channel without sticking to the inner channel walls. We consider that the choices of buffer, surfactant, and surface modification of particles and the developed cleaning process for quartz glass are important factors in the excellent particle-nanochannel control achieved in these experiments.
In Sect. 2.6, we comment on the data for electrostatic properties of the material in this study. However, the effect of hydrodynamic force and electrostatic forces between the particles and between the particle and the inner walls may be important for a complete understanding of nanoparticle motion. Since all-quartz-glass channels can provide uniform wall surface conditions compared with the one using PDMS, the nanofluidic device developed in this study may contribute to further detailed analysis in future, provided that the observation systems are improved, with higher sampling rate and finer spatial resolution using, e.g., evanescent wavebased particle velocimetry (Kazoe et al. 2015).

In this demonstration, we conclude that the laser irradiation at the entrance of the contraction can control the onset of particle translocation into the nanoslit. As in our previous study using a micro-scale contraction (Tsuji et al. 2018), such a flow switching can be used to enhance the performance of particle detection/identification in narrow space such as nanopore. To be more precise, we can combine a large external force, for example, a large pressure difference between the inlet and outlet, and the optical force to collect target particles effectively at the entrance. Then, we switch to a weak external force and stop the laser irradiation to achieve slow particle translocation into the contraction, where detecting sensors, such as nanogap electrodes, could be installed. In this way, we can get a high translocation frequency and high time resolution of reading signals at the same time.

Let us comment on some possible future work using the two-dimensional optical trapping in nanoslits. To elucidate the detailed mechanics during the optical trapping, it is necessary to evaluate the hydrodynamic stress acting on the particle confined in the nanoslit. The characteristics of hydrodynamic stress could be different from that of the particle trapped in the bulk, since the surface effect is expected to be prominent in our case. However, for the systematic evaluation of hydrodynamic stress, the precision of nanofabrication technique should be improved much more, so that the gaps between the particle surface and channel lid/bottom can be controlled. It is beyond the scope of the present research, and we leave this subject as a future subject.

\subsection{Three-dimensional clustering in nanoslit}

Here, we show the results of nanoparticles with diameter $d=190 \mathrm{~nm}$. The concentration of the particles in the working fluid is $10^{-4} \mathrm{wt} \%$. The experimental conditions are the same as those in Sect. 3.1, except for a different mirror unit (U-FBW, Olympus, Tokyo, Japan) designed for the observation of the fluorescence of particles with $d=190 \mathrm{~nm}$ (excitation: $505 \mathrm{~nm}$; emission: $515 \mathrm{~nm}$ ). These particles are expected to yield three-dimensional clusters in the nanoslit, because the nanoslit height can contain a couple of particles 
in the $z$ direction, that is, the height-to-diameter ratio for this case is $H / d \approx 3.2$. Because the scattering force pushes the particles toward the lid, the cluster is expected to be rather close to the lid.

Figure 9 shows snapshots of the observed particle motion in the test section for the case of $d=190 \mathrm{~nm}$. Note that Fig. 9 presents the magnification near the entrance of the contraction. The laser irradiation starts at $t=10 \mathrm{~s}$. The obvious difference between the case of $d=490 \mathrm{~nm}$ in Figs. 6 and 9 is that we cannot identify individual nanoparticles due to the smallness of the particle size and fluorescent halo. The particles start to form a cluster at $t=30 \mathrm{~s}$ near the laser focus, and the size of the cluster grows as time goes on. In the case of $d=190 \mathrm{~nm}$, we observe that several particles with trajectories close to the channel wall cannot be trapped optically. These particles can enter into the contraction regardless of the laser irradiation. Such an escape from trapping may be attributed to the smallness of the particle size, because the gradient force is proportional to the particle diameter cubed. Figure 10 presents an estimate of the number of clustered particles during the experiment. The estimation is calculated by converting the overall fluorescence intensity of the cluster into a particle count. It is seen that we can make a cluster containing almost 80 particles. Note that, if one tries to carry out the particle tracking analysis to investigate the dynamics of particles in the cluster, fluorescence intensity measurement may cause error due to halo and some localization technique should be introduced.

Figure 11 shows the translocation of particles with diameter $d=190 \mathrm{~nm}$. Here, we stop the laser irradiation at $t=0 \mathrm{~s}$. The cluster is released and the cloud of nanoparticles starts to enter the contraction channel at a speed of $44 \mu \mathrm{m} \mathrm{s}^{-1}$. This experiment demonstrates that

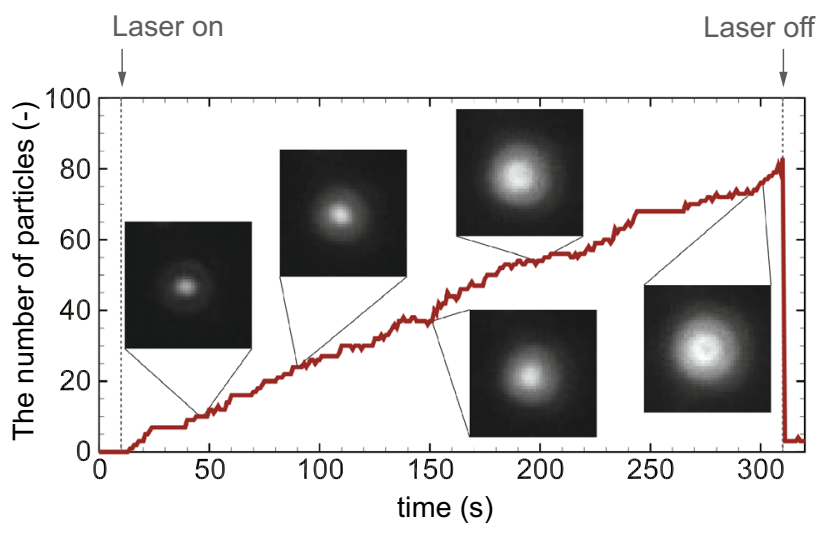

Fig. 10 Time development of the number of particles $N$ in the cluster for the case of $d=190 \mathrm{~nm}$

the translocation of particles with $d=190 \mathrm{~nm}$ can also be controlled using laser irradiation of the nanoslit channel. From the experimental results that we obtain, it seems that the trapped particles can withstand for the flow speed of about $50 \mu \mathrm{m} \mathrm{s}^{-1}$. However, it should be noted that the flow speed in the contraction channel may be dependent on the size of the cluster, since the cluster makes the effective cross-sectional area of contraction entrance smaller. Moreover, because the magnitude of optical force is the decreasing function of radial distance, the possibility of having escaping particle is dependent on the size of the cluster. Therefore, the precise estimation of the flow speed at which the trapped particle is released from the cluster is not carried out in the present paper, and will be our future work.
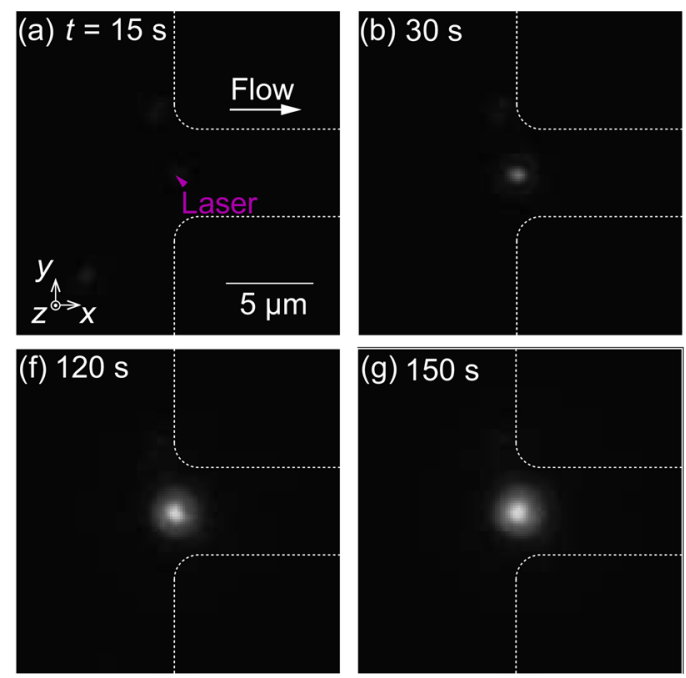
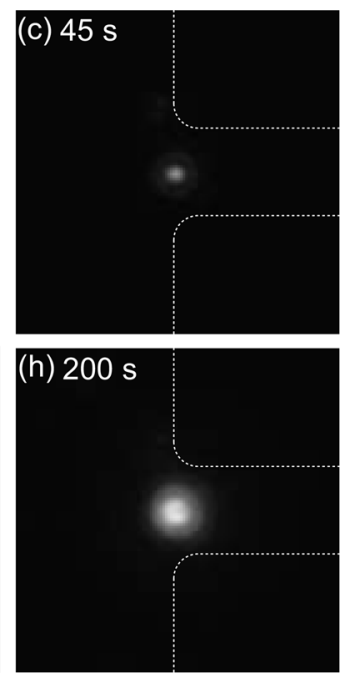
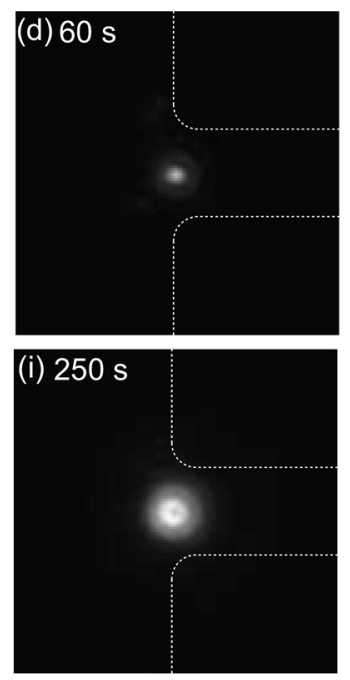
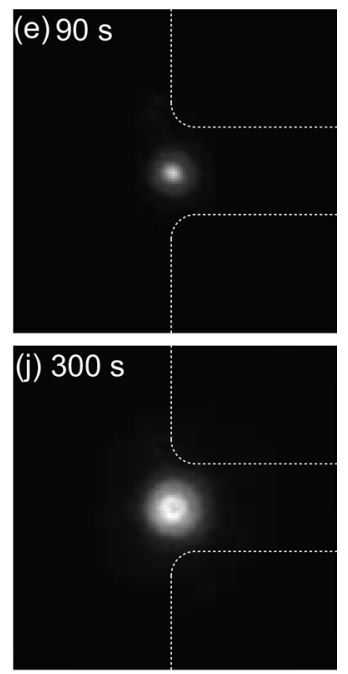

Fig. 9 Time series of the snapshots for the case of particles with $d=190 \mathrm{~nm}$ at $t=\mathbf{a} 15, \mathbf{b} 30, \mathbf{c} 45, \mathbf{d} 60$, e 90, f 120, g 150, h 200, i $250, \mathbf{j} 300 \mathrm{~s}$. The laser irradiation is incident at the center of each image, as shown in (a). The dashed lines outline the nanoslit pattern. The cluster of particles forms and grows as time goes on 


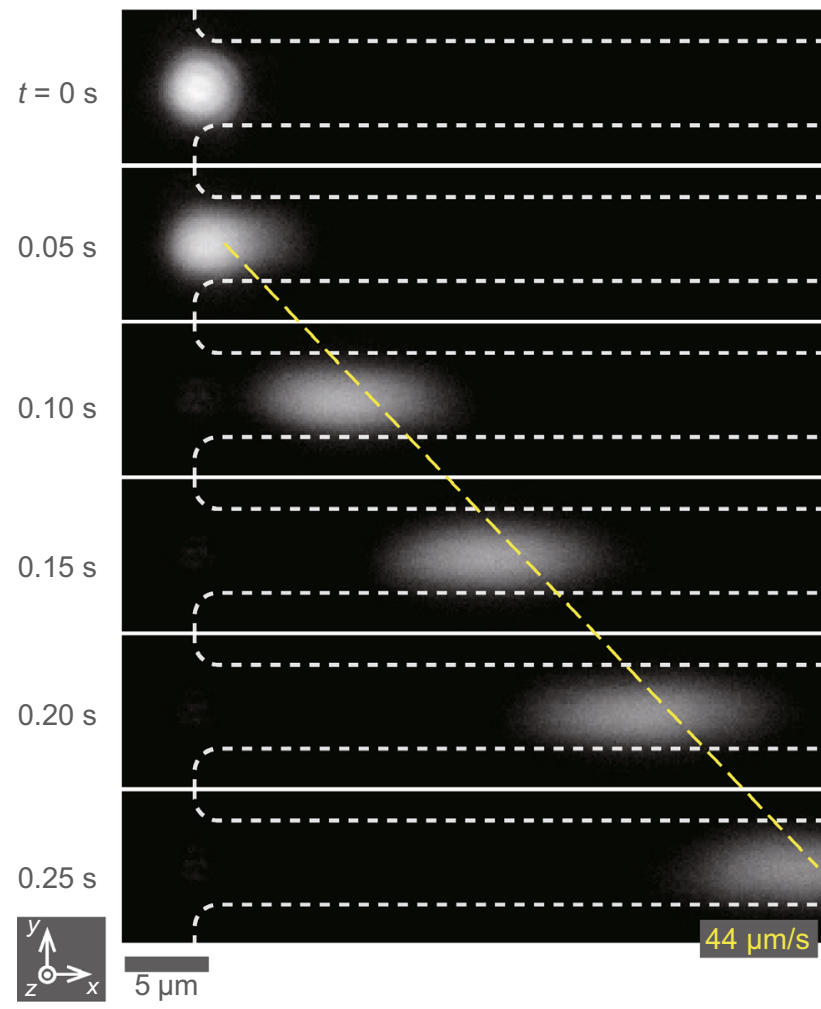

Fig. 11 Time series of the release of the cluster for $d=190 \mathrm{~nm}$. The laser irradiation is stopped at $t=0 \mathrm{~s}$, and the particles flow into the nanochannel as time goes on. The yellow dashed line indicates the particle flow speed of $44 \mu \mathrm{m} / \mathrm{s}$

Finally, we comment on the possibility of size-selective particle translocation. The magnitude of the optical gradient force for nanoparticle is proportional to the cube of the particle diameter, as discussed previously in this section. On the other hand, the drag force is proportional to the diameter. That is, the trapping of larger particles is more stable. Therefore, if the mixture of the particles with $d=190 \mathrm{~nm}$ and $490 \mathrm{~nm}$ is used as a sample, there is a high possibility to find the condition of the laser power or the flow rate, where only the particles with $d=490 \mathrm{~nm}$ are trapped and only the particles with $d=190 \mathrm{~nm}$ are translocated into the contraction channel.

\section{Conclusions}

In the present paper, we have proposed a method to control particle translocation into a contraction channel formed by a nanoslit using the technique of optical trapping by a focused near-infrared laser. The particle sizes tested in the experiments were $d=190$ and $490 \mathrm{~nm}$, which correspond to the effective diameters of viruses or allergen particles. The nanoparticles were successfully trapped at the entrance of the contraction channel by an optical force and subsequently released to enter into the contraction when the laser irradiation was stopped. In particular, for the case of $d=490 \mathrm{~nm}$, the trapped particles formed a two-dimensional cluster in the nanoslit. Two-dimensional clusters may be preferred to investigate the particle nature, such as size and inter-particle interaction, since the data analysis can be simplified by neglecting unobservable height direction, i.e., the $z$ direction. Such an optical control of the nanoparticle translocation into the contraction is expected to improve the functions of nanofluidic sensors, such as nanopore sequencers, by providing a tool to optimize the translocation frequency and speed. The flow control of biomaterial in the nanoslit channel is our future work.

Finally, we close the paper by giving some possible improvements of the present paper. The optical trapping of plasmonic nanoparticles, such as gold nanoparticle, has been demonstrated in the literature (Hansen et al. 2005; Hajizadeh and Reihani 2010), since these samples had large polarizability and were rather easy to be trapped. On the contrary, dielectric particles such as polystyrene or biomaterials have small polarizability and are still difficult to be stably trapped. Given such a situation, some non-standard optical trapping techniques, such as plasmonic optical tweezers (Shoji and Tsuboi 2014; Ndukaife et al. 2018; Pin et al. 2018) or thermophoretic manipulation induced by photothermal effects (Duhr and Braun 2006; Jiang et al. 2009; Maeda et al. 2012; Lin et al. 2018; Tsuji et al. 2018), have been proposed. These non-standard optical trapping techniques can be combined with the present flow control concept in nanofluidic device to achieve more stable performance.

Acknowledgements This research is supported by the Japan Society for the Promotion of Science (JSPS) KAKENHI Grant no. JP18H05242 for Scientific Research (S), JSPS KAKENHI Grant no. JP18K13687 for Young Scientists, and JSPS KAKENHI Grant no. JP16H06504 in Scientific Research on Innovative Areas, "Nano-Material Optical-Manipulation."

Open Access This article is distributed under the terms of the Creative Commons Attribution 4.0 International License (http://creativeco mmons.org/licenses/by/4.0/), which permits unrestricted use, distribution, and reproduction in any medium, provided you give appropriate credit to the original author(s) and the source, provide a link to the Creative Commons license, and indicate if changes were made.

\section{References}

Amini H, Lee W, Di Carlo D (2014) Inertial microfluidic physics. Lab Chip 14:2739-2761

Arima A, Harlisa IH, Yoshida T, Tsutsui M, Tanaka M, Yokota K, Tonomura W, Yasuda J, Taniguchi M, Washio T et al (2018) Identifying single viruses using biorecognition solid-state nanopores. J Am Chem Soc 140:16834-16841

Ashkin A (1970) Acceleration and trapping of particles by radiation pressure. Phys Rev Lett 24:156-159 
Ashkin A, Dziedzic JM, Bjorkholm JE, Chu S (1986) Observation of a single-beam gradient force optical trap for dielectric particles. Opt Lett 45:288-290

Bouvier NM, Palese P (2008) The biology of influenza viruses. Vaccine 26:D49

Briggs JAG, Grünewald K, Glass B, Förster F, Kräusslich H-G, Fuller SD (2006) The mechanism of HIV-1 core assembly: Insights from three-dimensional reconstructions of authentic virions. Structure $14: 15$

Bruus H (2007) Theoretical microfluidics. Oxford University Press, Oxford

Di Ventra M, Taniguchi M (2016) Decoding DNA, RNA and peptides with quantum tunnelling. Nat Nanotechnol 11:117

Duhr S, Braun D (2006) Why molecules move along a temperature gradient. Proc Natl Acad Sci USA 103:19678

Fukuyama T, Nakama S, Maeda YT (2018) Thermal molecular focusing: tunable cross effect of phoresis and light-driven hydrodynamic focusing. Soft Matter 14:5519-5524

Hajizadeh F, Reihani SNS (2010) Optimized optical trapping of gold nanoparticles. Opt Express 18:551-559

Hansen PM, Bhatia VK, Harrit N, Oddershede L (2005) Expanding the optical trapping range of gold nanoparticles. Nano Lett 5:1937-1942

Harada Y, Asakura T (1996) Radiation forces on a dielectric sphere in the rayleigh scattering regime. Opt Commun 124:529-541

Jiang H-R, Wada H, Yoshinaga N, Sano M (2009) Manipulation of colloids by a nonequilibrium depletion force in a temperature gradient. Phys Rev Lett 102:208301

Kawaguchi C, Noda T, Tsutsui M, Taniguchi M, Kawano S, Kawai $\mathrm{T}$ (2012) Electrical detection of single pollen allergen particles using electrode-embedded microchannels. J Phys Condens Matter 24:164202

Kazoe Y, Mawatari K, Kitamori T (2015) Behavior of nanoparticles in extended nanospace measured by evanescent wave-based particle velocimetry. Anal Chem 87:4087-4091

Keyser UF (2011) Controlling molecular transport through nanopores. J R Soc Interface 8:1369

Keyser UF, Van der Does J, Dekker C, Dekker NH (2006) Optical tweezers for force measurements on DNA in nanopores. Rev Sci Inst 77:105105

Kikuchi K, Mochizuki O (2011) Micro-PIV (micro particle image velocimetry) visualization of red blood cells (RBCs) sucked by a female mosquito. Meas Sci Technol 22:064002

Lin L, Wang M, Peng X, Lissek EN, Mao Z, Scarabelli L, Adkins E, Coskun S, Unalan HE, Korgel BA et al (2018) Opto-thermoelectric nanotweezers. Nat Photonics 12:195

Maeda YT, Tlusty T, Libchaber A (2012) Effects of long DNA folding and small RNA stem-loop in thermophoresis. Proc Natl Acad Sci USA 109:17972

Martel JM, Toner M (2014) Inertial focusing in microfluidics. Ann Rev Biomed Eng 16:371-396

Merchant CA, Healy K, Wanunu M, Ray V, Peterman N, Bartel J, Fischbein MD, Venta K, Luo Z, Johnson ATC et al (2010) DNA translocation through graphene nanopores. Nano Lett 10:2915
Nagura R, Tsujimura T, Tsuji T, Doi K, Kawano S (2019) Coarsegrained particle dynamics along helical orbit by an optical vortex irradiated in photocurable resins. OSA Continuum 2:400-415

Ndukaife JC, Xuan Y, Nnanna A, George A, Kildishev AV, Shalaev VM, Wereley ST, Boltasseva A (2018) High-resolution largeensemble nanoparticles trapping with multifunctional thermoplasmonic nanohole metasurface. ACS Nano 12:5376-5384

Nguyen NT, Wereley ST (2002) Fundamentals and applications of microfluidics. Artech House, Boston

Nito F, Shiozaki T, Nagura R, Tsuji T, Doi K, Hosokawa C, Kawano S (2018) Quantitative evaluation of optical forces by single particle tracking in slit-like microfluidic channels. J Phys Chem C 122:17963-17975

Pin C, Ishida S, Takahashi G, Sudo K, Fukaminato T, Sasaki K (2018) Trapping and deposition of dye-molecule nanoparticles in the nanogap of a plasmonic antenna. ACS Omega 3:4878-4883

Sackmann EK, Fulton AL, Beebe DJ (2014) The present and future role of microfluidics in biomedical research. Nature 507:181

Schneider GF, Kowalczyk SW, Calado VE, Pandraud G, Zandbergen HW, Vandersypen LMK, Dekker C (2010) DNA translocation through graphene nanopores. Nano Lett 10:3163

Shoji T, Tsuboi Y (2014) Plasmonic optical tweezers toward molecular manipulation: tailoring plasmonic nanostructure, light source, and resonant trapping. J Phys Chem Lett 5:2957-2967

Streletzky K, Phillies GDJ (1995) Temperature dependence of Triton X-100 micelle size and hydration. Langmuir 11:1

Tanaka S, Tsutsui M, Theodore H, Yuhui H, Arima A, Tsuji T, Doi K, Kawano S, Taniguchi M, Kawai T (2016) Tailoring particle translocation via dielectrophoresis in pore channels. Sci Rep 6:31670

Tsuji T, Kozai K, Ishino H, Kawano S (2017) Direct observations of thermophoresis in microfluidic systems. Micro Nano Lett 12:520

Tsuji T, Saita S, Kawano S (2018) Dynamic pattern formation of microparticles in a uniform flow by an on-chip thermophoretic separation device. Phys Rev Appl 9:024035

Tsuji T, Sasai Y, Kawano S (2018) Thermophoretic manipulation of micro- and nanoparticle flow through a sudden contraction in a microchannel with near-infrared laser irradiation. Phys Rev Appl 10:044005

Tsutsui M, Taniguchi M, Yokota K, Kawai T (2010) Identifying single nucleotides by tunnelling current. Nat Nanotechnol 5:286

Wang C, Xu J, Guo S, Kang Q, Wang Y, Wang Y, Tian Y (2019) A facile method for direct bonding of single-crystalline $\mathrm{SiC}$ to $\mathrm{Si}, \mathrm{SiO}_{2}$, and glass using VUV irradiation. Appl Surface Sci 471:196-204

Xu Y (2017) Nanofluidics: a new arena for materials science. Adv Mater 1702419:1-17

Yusko EC, Johnson JM, Majd S, Prangkio P, Rollings RC, Li J, Yang J, Mayer M (2011) Controlling protein translocation through nanopores with bio-inspired fluid walls. Nat Nanotechnol 6:253

Publisher's Note Springer Nature remains neutral with regard to jurisdictional claims in published maps and institutional affiliations. 